solv-int/9811015

\title{
Nonlinear Evolution Equations Invariant Under Schrödinger Group in three-dimensional Space-time
}

\author{
F. GÜNGÖR \\ Department of Mathematics, Faculty of Science \\ Istanbul Technical University \\ 80626, Istanbul, Turkey \\ gungorf@itu.edu.tr
}

\begin{abstract}
A classification of all possible realizations of the Galilei, Galilei-similitude and Schrödinger Lie algebras in three dimensional space-time in terms of vector fields under the action of the group of local diffeomorphisms of the space $\mathbb{R}^{3} \times$ $\mathbb{C}$ is presented. Using this result a variety of general second order evolution equations invariant under the corresponding groups are constructed and their physical significance are discussed.
\end{abstract}

\section{Introduction}

A useful tool for treating nonlinear differential equations is the symmetry method. This includes, among others, generating a family of nontrivial exact solutions from a known (trivial) one, reducing the order for ODEs and the number of independent variables for PDEs and classifying equations into equivalence classes and hence deriving the necessary conditions for apparently different equations to be transformable amongst each other. This method has been used to obtain exact particular solutions of a large number of physically important nonlinear partial differential equations. On the other hand, if we know the symmetry group of an equation we can construct other equations which are more general and left invariant by the same group. The great advantage of this inverse approach is that symmetry reduction technique can be used 
to find some exact solutions of a large class of equations which are by construction invariant.

In spite of the abundance of work pursuing the direct method (determining symmetry group and then finding particular solutions of the reduced equations), there appear only a few papers devoted to the alternative problem of constructing the most general equations invariant under a given symmetry group [1, 2, 3, 4, 5]. Surprisingly, the complete classification of invariant equations for many of the groups of physical importance with arbitrary spatial dimensions is unknown. Though in [5] the most general second order invariant equations for the rotation group $\mathrm{O}(n)$ as well as the Euclidean, Poincarè and conformal groups are constructed, this approach seems cumbersome and less systematic. We mention that higher dimensional invariant equations for the above groups is also unknown. A more systematic way of constructing general equations invariant under a group is adopted in [1, 3]. An extension of the results of [1] to a higher dimensional case was carried out in [2].

Recently, there has been an explosion of papers devoted to a search for modified versions of quantum mechanics in which the superposition principle is no longer valid and the evolution equation is no longer linear. For instance, Sabatier [6] introduced a set of nonlinear equations of the Schrödinger type. In particular, he studied two classes of modular equations depending on a real parameter. Later, Auberson and et al [7] showed that these equations can be linearized by scaling variables. It turned out that only a subclass of this set, i.e. the so called modular class for the free particle case shares the property of being norm conserving and being homogeneous, time reversal invariant with the linear Schrödinger equation. Another remarkable fact is that, besides being Galilei invariant as mentioned in [7], they have the same invariance group as the linear Schrödinger equation. In the meanwhile, Doebner and Goldin 8] proposed an eight parameter family of homogeneous nonlinear Schrödinger type equations on $\mathbb{R}^{3}$, including a diffusion term, called DG-equations from fundamental considerations of local symmetry in quantum mechanics. They possess nice symmetry properties; for certain choices of the five parameters they are invariant under a central extension of the Galilei group. A study of symmetries of the free DG-equations in terms of gauge invariants and the integrability of certain sub-families associated with their symmetries has been reviewed in [9]. As a matter of fact DG-equations include numerous modifications of the linear Schrödinger equation. For example, equations studied in [6] are special cases of DG-equations. One observation that occurs in equations proposed as a possible modification of the Schrödinger equation from physical considerations only is that they generally do not obey the Galilean principle. On the other hand, Galilei invariant equations are usually consistent with dilation and conformal invariance. That is why a group-theoretical justification for this modification is of vital importance. From this point of view the construction of all nonlinear evolution type equations preserving Schrödinger invariance will play an essential role 
in modifying Schrödinger equation. Let us emphasize that on physical grounds the property of homogeneity is an important ingredient in nonlinear Schrödinger equations. Indeed, Weinberg [10] proposed tests of quantum mechanics by modifying the Schrödinger equation by nonlinear terms and then imposing a complex homogeneity condition. One can formulate such a theory by requiring that the fundamental equations be invariant under a group isomorphic to the symmetry group of the linear Schrödinger equation, but omitting the superposition principle.

It is the objective of this paper to construct the nonlinear second order evolution equations

$$
\psi_{t}+F\left(x, y, t, \psi, \psi^{*}, \psi_{i}, \psi_{i}^{*}, \psi_{i j}, \psi_{i j}^{*}\right)=0, \quad i, j \in\{x, y\} .
$$

invariant under the Galilei, Galilei-similitude and Schrödinger groups. In (1.1), $\psi(x, y, t)$ is a complex function, the star denotes complex conjugation, the subscripts denote spatial derivatives and $F$ is a complex function of the indicated variables. In particular, linear Schrödinger equation for a free particle

$$
i \psi_{t}+\Delta \psi=0
$$

where $\Delta$ is the Laplace operator in two dimensional Euclidean space, is contained in the set of equations (1.1). It is easy to show that (1.2) admits nine dimensional Schrödinger group and an infinite dimensional invariant subgroup reflecting the linearity of the equation (linear superposition principle). An additional symmetry corresponds to the homogeneity property stating that if $\psi$ is a solution to (1.2) then so is $\alpha \psi$ where $\alpha$ is a complex constant. A similar problem for $\operatorname{Sch}(1)$ is solved in [3] and a new realization of $\operatorname{Sch}(1)$ completing the results of [3] is given in [11]. Fushchich et al 12] constructed systems of $(n+1)$-dimensional quasilinear second order evolution equations invariant under $\mathfrak{s c h}(n)$. They first make an ansatz about the form of the equation as a reasonable generalization of a linear equation and then impose invariance.

The organization of the paper is as follows. In section 2, as a first step towards classifying Schrödinger invariant equations, we classify all possible inequivalent realizations of the Galilei $\mathfrak{g}(2,1)$, Galilei-similitude $\mathfrak{s g}(2,1)$ and Schrödinger $\mathfrak{s c h}(2)$ Lie algebras in terms of vector fields under the action of local diffeomorphisms of the space $\mathbb{R}^{3} \times \mathbb{C}$. In section 3 , we obtain second order differential invariants of the realized vector fields and hence second order $\mathfrak{s c h}(2)$ invariant differential equations. We also identify $\mathfrak{s c h}(2)$ invariant equations satisfying homogeneity condition. We conclude the paper with some remarks and a summary of the results obtained. Finally, let us emphasize that throughout the paper invariance under a symmetry group will be in the sense that the equation is annihilated by second order prolongations of the vector fields on the solution set. Certainly, this invariance requirement is more restricted 
than the case imposed for relativistic equations [1, 2]. In the present article we shall not take into account the infinite dimensional Lie group reflecting superposition principle of all linear differential equations.

\section{Realizations of the Lie algebras by vector fields}

\subsection{Schrödinger group $\operatorname{Sch}(2)$ and its Lie algebra}

The Schrödinger group Sch(2) is nine parameters local group of transformations of the space $\mathbb{R}^{3} \times \mathbb{C}$. It is a Lie group isomorphic to

$$
H_{2} \triangleright\{\mathrm{SL}(2, \mathbb{R}) \otimes \mathrm{SO}(2)\}
$$

where $\triangleright$ denotes a semi-direct product and $\mathrm{SL}(2, \mathbb{R}), \mathrm{SO}(2)$ and $\mathrm{H}_{2}$ are the special linear group, rotation group in the plane and Heisenberg group, respectively. It is also subgroup of the conformal group $\mathrm{O}(4,2)$ of a $(2+1)$-dimensional Minkowski space $\mathrm{M}(2,1)$ which leaves the Hamilton-Jacobi equation in two space dimensions invariant [2]. Schrödinger group appears to be symmetry group of a variety of physically significant equations. Indeed, we already mentioned that (1.2) is invariant under $\mathrm{Sch}(2)$. It is isomorphic to the symmetry group of two-dimensional heat equation. It is also symmetry group of the 2-dimensional Navier-Stokes equations in the case when a linear homogeneous transformation law is imposed on the pressure.

A recent study of finite dimensional nonrelativistic conformal groups is given in 13.

The corresponding Lie algebra has a basis spanned by two space translations $P_{1}, P_{2}$, two Galilei boosts $B_{1}, B_{2}$, translation of the phase $M$, time translation $T$, dilation $D$, rotation $J$ and conformal transformations $C$. This means that we are dealing with a nine dimensional Lie algebra $\mathfrak{s c h}(2)$ which can be written as a semidirect sum reflecting the Levi decomposition

$$
\begin{aligned}
\mathfrak{s c h}(2) & =\mathfrak{h}_{2} \square\{\operatorname{sl}(2, \mathbb{R}) \oplus \operatorname{so}(2)\} \\
& \sim\left\{P_{1}, P_{2}, B_{1}, B_{2}, M\right\} \square\{T, C, D, J\}
\end{aligned}
$$

with nonzero commutation relations

$$
\begin{array}{lll}
{\left[P_{1}, B_{1}\right]=M / 2,} & {\left[P_{2}, B_{2}\right]=M / 2,} & \\
{\left[J, B_{2}\right]=-B_{1},} & {\left[J, P_{2}\right]=-P_{1},} & \\
{\left[J, B_{1}\right]=B_{2},} & {\left[J, P_{1}\right]=P_{2},} & \\
{\left[T, B_{j}\right]=P_{j},} & {\left[D, B_{j}\right]=B_{j}, \quad j=1,2} & \\
{\left[D, P_{j}\right]=-P_{j},} & {\left[C, P_{j}\right]=-B_{j}, \quad j=1,2} & \\
{[T, D]=2 T,} & {[T, C]=D,} & {[D, C]=2 C .}
\end{array}
$$


In (2.2), $\mathfrak{h}_{2} \sim\left\{P_{1}, P_{2}, B_{1}, B_{2}, M\right\}$ is the Heisenberg algebra with center $M$. The subalgebra

$$
\left\{P_{1}, P_{2}, T, B_{1}, B_{2}, J, M\right\}
$$

corresponds to the extended Galilei algebra, and subalgebra

$$
\left\{D, P_{1}, P_{2}, T, B_{1}, B_{2}, J, M\right\}
$$

to the extended Galilei-similitude algebra. Let us remark that actually different Levi decompositions of $\mathfrak{s c h}(2)$ exist [14].

The action of the symmetry group on the space $\left(x, y, t, \psi, \psi^{*}\right)$ is obtained by integrating the vector fields of (2.2) which is referred to as exponentiation of the vector fields.

\subsection{Realizations of Galilei, Galilei-similitude and Schrödinger algebras by vector Fields}

We classify realizations of $\mathfrak{g}(2,1), \mathfrak{s} \mathfrak{g}(2,1)$ and $\mathfrak{s c h}(2)$ in terms of vector fields of the form

$$
\mathbf{v}=\xi \partial_{x}+\eta \partial_{y}+\tau \partial_{t}+\sigma \partial_{\psi}+\sigma^{*} \partial_{\psi^{*}}
$$

where $\xi, \eta, \tau, \sigma, \sigma^{*}$ are functions of $x, y, t, \psi, \psi^{*}$ with $\xi, \eta, \tau \in \mathbb{R}$, and $\sigma, \sigma^{*} \in \mathbb{C}$, under local diffeomorphisms of the space $\mathbb{R}^{3} \times \mathbb{C}$ parametrized by the variables $(x, y, t, R, \phi)$ with $x, y, t$ space-time coordinates and $\psi=R e^{i \phi}$ the wave function. Two realizations will be equivalent if the corresponding vector fields can be transformed into each other by arbitrary smooth invertible changes of the independent and dependent variables:

$$
\begin{array}{ll}
\tilde{x}=X(x, y, t, R, \phi), & \tilde{y}=Y(x, y, t, R, \phi), \quad \tilde{t}=T(x, y, t, R, \phi) \\
\tilde{R}=\Psi(x, y, t, R, \phi), & \tilde{\phi}=\Phi(x, y, t, R, \phi) .
\end{array}
$$

\subsubsection{The extended Galilei algebra}

We start from the four-dimensional abelian algebra $\left\{P_{1}, P_{2}, T, M\right\}$. It is immediate to rectify these vector fields (see [15, 16] for the vector field rectification theorem) up to the diffeomorphisms as

$$
\mathcal{A}_{4}:\left\{P_{1}=\partial_{x}, P_{2}=\partial_{y}, T=\partial_{t}, M=\partial_{\phi}\right\}
$$

$\mathcal{A}_{4}$ generates space-time translations and translation of phase of the wave function. Obviously, these vector fields remain invariant under

$$
\begin{gathered}
\tilde{x}=x+g(R), \quad \tilde{y}=y+h(R), \quad \tilde{t}=t+f(R), \\
\tilde{\phi}=\phi+\Phi(R), \quad \tilde{R}=\rho(R)
\end{gathered}
$$


where $f, g, h, \Phi$ and $\rho$ are arbitrary functions of $R$. Let us mention that the standard vector-field realizations in $2+1$ dimensional space-time can be found in [17]. If the Galilei boost $B_{1}$ having the form (2.4) is subjected to the commutation relations involving $B_{1}$ and elements of the already realized algebra $\mathcal{A}_{4}$ and is further simplified by (2.7), one finds precisely two inequivalent types of realizations of $B_{1}$ :

$$
\begin{aligned}
& B_{1}^{(1)}=t \partial_{x}+f_{2}(R) \partial_{y}+f_{3}(R) \partial_{t}+x / 2 \partial_{\phi} \\
& B_{1}^{(2)}=t \partial_{x}+R \partial_{R}+x / 2 \partial_{\phi} .
\end{aligned}
$$

Next we realize $B_{2}$ written in the form (2.4). Imposing commutation relations and simplifying by transformations leaving the algebra $\left\{\mathcal{A}_{4}, B_{1}^{(j)}, \quad j=1,2\right\}$ invariant it follows that $B_{1}$ can be further extended and we have

$$
\begin{aligned}
& B_{1}^{(1)}=t \partial_{x}+f_{2}(R) \partial_{y}+x / 2 \partial_{\phi} \\
& B_{2}^{(1)}=f_{2}(R) \partial_{x}+\left(t+g_{2}(R)\right) \partial_{y}+y / 2 \partial_{\phi}
\end{aligned}
$$

and

$$
\begin{aligned}
& B_{1}^{(2)}=t \partial_{x}+R \partial_{R}+x / 2 \partial_{\phi} \\
& B_{2}^{(2)}=t \partial_{y}+\mu R \partial_{R}+y / 2 \partial_{\phi}
\end{aligned}
$$

where $\mu$ is a constant. Proceeding as above we see that the extension of $\left\{\mathcal{A}_{4}, B_{1}^{(2)}, B_{2}^{(2)}\right\}$ to rotations is not possible. However, for (2.9a)-(2.9b) we find

$$
J=y \partial_{x}-x \partial_{y}+j_{3}(R) \partial_{t}+j_{4}(R) \partial_{R}+j_{5}(R) \partial_{\phi}
$$

with $f_{2}$ and $g_{2}$ related by

$$
4 f_{2} f_{2}^{\prime}+g_{2} g_{2}^{\prime}=0
$$

and $j_{3}=2 f_{2}, j_{4}, j_{5}$ arbitrary. Consequently, we obtain a single realization of the Galilei algebra with infinitesimal generators (2.6), (2.9a)-(2.9b) and (2.10) depending on three arbitrary functions.

\subsubsection{The extended Galilei-similitude algebra}

Let us now add a dilation generator $D$ of the form (2.4). The commutation relations between $\left\{P_{1}, P_{2}, B_{1}, B_{2}, T, M, D\right\}$ restricts $D$ to

$$
D=x \partial_{x}+y \partial_{y}+2 t \partial_{t}+d_{4}(R) \partial_{R}+d_{5}(R) \partial_{\phi} .
$$

Commuting $J$ with $D$ yields

$$
j_{4}(R)=a d_{4}(R) \quad \text { and } \quad j_{5}(R)=a d_{5}(R), \quad a=\text { const. }
$$


and forces $B_{1}^{(1)}, B_{2}^{(1)}$ and $J$ to be

$$
\begin{gathered}
B_{1}^{(1)}=t \partial_{x}+x / 2 \partial_{\phi}, \quad B_{2}^{(1)}=t \partial_{y}+y / 2 \partial_{\phi}, \\
J=y \partial_{x}-x \partial_{y}+a\left(d_{4}(R) \partial_{R}+d_{5}(R) \partial_{\phi}\right) .
\end{gathered}
$$

The form of $D$ is further restricted by transformations leaving the algebra

$$
\left\{P_{1}, P_{2}, B_{1}, B_{2}, M, T, J\right\}
$$

invariant. Finally, we obtain two inequivalent realizations of the Galilei-similitude algebra which depend on one arbitrary function

$$
\begin{gathered}
s g^{1}\left(d_{0}\right): \begin{cases}B_{1} & =t \partial_{x}+x / 2 \partial_{\phi}, \quad B_{2}=t \partial_{y}+y / 2 \partial_{\phi} \\
J & =y \partial_{x}-x \partial_{y}-a R / 2 \partial_{R}+a d_{0}(R) \partial_{\phi} \\
D & =x \partial_{x}+y \partial_{y}+2 t \partial_{t}-R / 2 \partial_{R}+d_{0}(R) \partial_{\phi}\end{cases} \\
s g^{2}\left(\delta_{0}\right): \begin{cases}B_{1} & =t \partial_{x}+x / 2 \partial_{\phi}, \quad B_{2}=t \partial_{y}+y / 2 \partial_{\phi} \\
J & =y \partial_{x}-x \partial_{y}+a \delta_{0}(R) \partial_{\phi} \\
D & =x \partial_{x}+y \partial_{y}+2 t \partial_{t}+\delta_{0}(R) \partial_{\phi} .\end{cases}
\end{gathered}
$$

\subsubsection{The Schrödinger algebra}

The above obtained realizations of $\mathfrak{s g}(2,1)$ can be further extended to $\mathfrak{s c h}(2)$ by adding the nonrelativistic conformal generator $C$. We start from (2.12) and repeat the routine steps of imposing commutation relations and simplifying by transformations leaving the $\mathfrak{s g}(2,1)$ algebra realizations unchanged. Omitting the lengthy details we present 
the final results only:

$$
\begin{gathered}
\operatorname{sch}^{1}(f ; \alpha, \beta):\left\{\begin{aligned}
J= & y \partial_{x}-x \partial_{y} \\
D= & x \partial_{x}+y \partial_{y}+2 t \partial_{t}-\frac{R}{2} \partial_{R}+f(R) \partial_{\phi} \\
C= & x t \partial_{x}+y t \partial_{y}+t^{2} \partial_{t}+\left(-\frac{t R}{2}+\alpha R^{-3}\right) \partial_{R} \\
& +\left(\left(x^{2}+y^{2}\right) / 4+t f(R)+R^{-4}(\beta-2 \alpha f(R))\right) \partial_{\phi}
\end{aligned}\right. \\
\operatorname{sch}^{2}(g):\left\{\begin{aligned}
J= & y \partial_{x}-x \partial_{y}-\frac{a R}{2} \partial_{R}+a g(R) \partial_{\phi} \\
D= & x \partial_{x}+y \partial_{y}+2 t \partial_{t}-\frac{R}{2} \partial_{R}+g(R) \partial_{\phi} \\
C= & x t \partial_{x}+y t \partial_{y}+t^{2} \partial_{t}-\frac{t R}{2} \partial_{R}+\left(\left(x^{2}+y^{2}\right) / 4+t g(R)\right) \partial_{\phi}
\end{aligned}\right. \\
\operatorname{sch}^{3}(h):\left\{\begin{aligned}
J= & y \partial_{x}-x \partial_{y}+a h(R) \partial_{\phi} \\
D= & x \partial_{x}+y \partial_{y}+2 t \partial_{t}+h(R) \partial_{\phi} \\
C= & x t \partial_{x}+y t \partial_{y}+t^{2} \partial_{t}+\left(\left(x^{2}+y^{2}\right) / 4+t h(R)\right) \partial_{\phi} .
\end{aligned}\right.
\end{gathered}
$$

We observe that all of the above realizations characterize a class of algebras corresponding to an arbitrary function and constants and generate fiber-preserving transformations. Using the relations

$$
R \partial_{R}=\psi \partial_{\psi}+\psi^{*} \partial_{\psi^{*}}, \quad \partial_{\phi}=i\left(\psi \partial_{\psi}-\psi^{*} \partial_{\psi^{*}}\right)
$$

we can express all the generators involving $R$ and $\phi$ (modulus and phase of the wave) in terms of the original variables $x, y, t, \psi, \psi^{*}$.

\section{Differential invariants and invariant equations}

In this section we obtain second order differential invariants under the particular cases of the realizations obtained in section 2.2 and hence invariant differential equations admitting Schrödinger symmetry.

A brief review of the differential invariants and fundamental theorem that is essential for constructing invariant equations was outlined in [2]. For a full discussion of the relevant definitions and theorems with proofs we refer to the contemporary literature 115, 16, 18, 19. In order to make the present paper self-contained we recall some definitions which will be needed in the derivation of differential invariants. Let 
$\mathrm{G}$ be a local Lie group of transformations acting on the space of independent and dependent variables $X \otimes U$ and $\mathrm{G}^{(n)}=\operatorname{pr}^{(n)} \mathrm{G}$ denote the prolonged group action on the jet space $J^{n}$ whose coordinates are denoted by $\left(x, u^{(n)}\right)$. The space of infinitesimal generators of G, i.e. its Lie algebra will be denoted by $\mathfrak{g}$ with associated prolongation $\mathfrak{g}^{(n)}=\operatorname{pr}^{(n)} \mathfrak{g}$. Recall that an absolute differential invariant of order $r \leq n$ is a scalar function $I: J^{n} \rightarrow \mathbb{R}$ which satisfies

$$
I\left(g^{(n)} .\left(x, u^{(n)}\right)\right)=I\left(x, u^{(n)}\right)
$$

for all $g^{(n)} \in \mathrm{G}^{(n)}$ and all $\left(x, u^{(n)}\right) \in J^{n}$. Since any function $F\left(I_{1}, I_{2}, \ldots I_{r}\right)$ of a collection of differential invariants $\left\{I_{1}, I_{2}, \cdots, I_{r}\right\}$ is also a differential invariant, we classify differential invariants up to functional independence. A complete set of functionally independent differential invariants will be called fundamental invariants. Once we have found such a complete set, any other differential invariant can be expressed as a function of these invariants. Let $\mathbf{v}$ be a one-parameter group of transformations acting on $X \otimes U$, the associated $n$-th order prolonged vector field $\operatorname{pr}^{(n)} \mathbf{v}$ is the vector field on the jet space $J^{n}$. The infinitesimal version of (3.1) can be written as

$$
\operatorname{pr}^{(n)} \mathbf{v}(I)=0
$$

for every prolonged vector field $\mathrm{pr}^{(n)} \mathbf{v}$. This implies that if $\left\{\mathbf{v}_{i}\right\}, i=1,2, \ldots, r$ form a basis for the symmetry algebra then the differential invariants are found by solving an overdetermined system of homogeneous, first order linear partial differential equations of (3.2) with $\mathbf{v}$ replaced by the basis elements $\mathbf{v}_{i}$. A solution to this system is a differential function depending on $n$-th order jet variables. One way to proceed towards finding differential invariants is to first solve the system for $\mathbf{v}_{1}$ which amounts to obtaining the differential invariants of $\mathbf{v}_{1}$ and next to re-express the remaining vector fields in terms of these invariants as coordinates and to find invariants of $r-1$ vector fields, namely to solve inductively the rest of the system containing $r-1$ equations using the same procedure. The general formula for the $n$-th prolongation of a vector field is given, e.g. in [15]. Since we concentrate on second order differential equations here, we need expressions for second prolongation $\operatorname{pr}^{(2)} \mathbf{v}_{i}$ for each infinitesimal generator $\mathbf{v}_{i}$. The most drudgery part of the present paper, i.e., the calculation of the explicit expressions for $\operatorname{pr}^{(2)} \mathbf{v}_{i}$ has been eliminated using MATHEMATICA.

The general form of an invariant evolution equation will be

$$
\psi_{t}+F\left(I_{1}, \cdots, I_{k}\right)=0
$$

where $\left\{I_{1}, \cdots, I_{k}\right\}$ are fundamental invariants. The function $F$ will be obtained as a solution to the partial differential equations

$$
\operatorname{pr}^{(2)} \mathbf{v}_{i}\left(\psi_{t}+F\right)=0, \quad \text { whenever } \quad \psi_{t}=-F
$$


for every vector field $\mathbf{v}_{i}$ in the basis and $F$ has a priori the form in (1.1). In other words, each element chosen from the realized algebra provides a first order linear partial differential equation for the function $F$. Solving this overdetermined system we express $F$ in terms of fundamental invariants whenever possible and hence construct invariant equations. In the following we apply this scheme to obtain the Galilei, Galilei-similitude and Schrödinger invariant equations, respectively.

\subsection{The extended Galilei invariant equations}

Consider the standard realization

$$
\begin{aligned}
\left\{\left\{\mathcal{A}_{4}\right\}, B_{1}\right. & =t \partial_{x}+\frac{i x}{2}\left(\psi \partial_{\psi}-\psi^{*} \partial_{\psi^{*}}\right), B_{2}=t \partial_{y}+\frac{i y}{2}\left(\psi \partial_{\psi}-\psi^{*} \partial_{\psi^{*}}\right) \\
J & \left.=y \partial_{x}-x \partial_{y}\right\}
\end{aligned}
$$

Invariance under $\mathcal{A}_{4}$ will restrict the form of (1.1) to

$$
\begin{array}{ll}
\psi_{t}+\psi_{x x}+F\left(I_{j}\right) \psi=0, & j=1,11 \\
I_{1}=\psi \psi^{*}=|\psi|^{2}, & \\
I_{2}=\psi^{*} \psi_{x}, & I_{3}=\psi \psi_{x}^{*}=I_{2}^{*} \\
I_{4}=\psi^{*} \psi_{x x}, & I_{5}=\psi \psi_{x x}^{*}=I_{4}^{*} \\
I_{6}=\psi^{*} \psi_{y}, & I_{7}=\psi \psi_{y}^{*}=I_{6}^{*} \\
I_{8}=\psi^{*} \psi_{y y}, & I_{9}=\psi \psi_{y y}^{*}=I_{8}^{*} \\
I_{10}=\psi^{*} \psi_{x y}, & I_{11}=\psi \psi_{x y}^{*}=I_{10}^{*} .
\end{array}
$$

A further requirement of invariance under $B_{1}$ reduces eleven invariants to ten and (3.4) to

$$
\begin{array}{ll}
\qquad i \psi_{t}+\psi_{x x}+F\left(J_{k}\right) \psi=0, & k=1,10 \\
& J_{2}=I_{2}+I_{3}=\left(|\psi|^{2}\right)_{x} \\
J_{1}=I_{1}, & J_{4}=I_{2}^{2}-I_{1} I_{4}=J_{3}^{*} \\
J_{3}=I_{3}^{2}-I_{1} I_{5}, & J_{\mu}=I_{\mu}, \quad \mu=6,7,8,9 \\
J_{5}=I_{1} I_{10}+I_{6} I_{3}, &
\end{array}
$$

Imposing invariance under $B_{2}$ will reduce ten invariants to nine and (3.5) to

$$
i \psi_{t}+\Delta \psi+F\left(K_{\lambda}\right) \psi=0, \quad \lambda=1,9
$$




$$
\begin{array}{ll}
K_{\sigma}=J_{\sigma}, \quad \sigma=1,2,3,4 & \\
K_{5}=J_{5}+J_{10}, & K_{6}=J_{6}+J_{7}=\left(|\psi|^{2}\right)_{y} \\
K_{7}=J_{6}^{2}-J_{1} J_{9}, & K_{8}=J_{7}^{2}-J_{1} J_{9}=K_{7}^{*} \\
K_{9}=J_{5}-J_{2} J_{6} . &
\end{array}
$$

where $K_{1}, K_{2}, K_{5}$ and $K_{6}$ are real. Finally if we require (3.6) be rotationally invariant we are led to the following extended Galilei-invariant equation

$$
\begin{array}{ll} 
& i \psi_{t}+\Delta \psi+F\left(L_{1}, L_{2}, L_{3}, L_{4}, L_{5}\right) \psi=0 \\
L_{1}=K_{1}, & L_{2}=K_{2}^{2}+K_{6}^{2} \\
L_{3}=K_{3}+K_{8}, & L_{4}=K_{4}+K_{7}=L_{3}^{*} \\
L_{5}=K_{9}^{2}-K_{4} K_{7} . &
\end{array}
$$

where $L_{1}$ and $L_{2}$ are real. In terms of the original variables we have

$$
\begin{aligned}
& L_{1}=|\psi|^{2} \\
& L_{2}=\left\{\left(|\psi|^{2}\right)_{x}\right\}^{2}+\left\{\left(|\psi|^{2}\right)_{y}\right\}^{2}=\left(\nabla|\psi|^{2}\right)^{2}=4|\psi|^{2}(\nabla|\psi|)^{2} \\
& L_{3}=\psi^{2}\left(\psi_{x}^{* 2}+\psi_{y}^{* 2}\right)-|\psi|^{2} \psi \Delta \psi^{*}=\psi^{2}\left(\nabla \psi^{*}\right)^{2}-|\psi|^{2} \psi \Delta \psi^{*} \\
& L_{4}=L_{3}^{*}=\psi^{* 2}\left(\psi_{x}^{2}+\psi_{y}^{2}\right)-|\psi|^{2} \psi^{*} \Delta \psi=\psi^{* 2}(\nabla \psi)^{2}-|\psi|^{2} \psi^{*} \Delta \psi \\
& L_{5}=\left\{|\psi|^{2}\left(\psi^{*} \psi_{x y}+\psi_{y} \psi_{x}^{*}\right)-\psi^{*} \psi_{y}|\psi|_{x}^{2}\right\}^{2}-\psi^{* 4}\left(\psi_{x}^{2}-\psi \psi_{x x}\right)\left(\psi_{y}^{2}-\psi \psi_{y y}\right)
\end{aligned}
$$

In particular, (3.7) includes a physically relevant subclass of nonlinear equations of the form

$$
i \psi_{t}+\Delta \psi+f(|\psi|) \psi=0
$$

\subsection{The extended Galilei-similitude invariant equations}

We now add the requirement that (3.7) be invariant under the dilation generator

$$
D_{1}=x \partial_{x}+y \partial_{y}+2 t \partial_{t}-2 / k\left(\psi \partial_{\psi}+\psi^{*} \partial_{\psi^{*}}\right) \quad k \neq 0
$$

which is equivalent to $D$ of (2.13a) by a simple change of variable with $f=0$ and obtain the invariant equation

$$
\begin{gathered}
i \psi_{t}+\Delta \psi+L_{1}^{k / 2} F\left(R_{1}, R_{2}, R_{3}\right) \psi=0 \\
R_{1}=\frac{L_{3}}{L_{1}^{(k+4) / 2}}, \quad R_{2}=\frac{L_{2}}{L_{1}^{(k+4) / 2}}, \quad R_{3}=\frac{L_{4}}{L_{1}^{(k+4) / 2}}=R_{1}^{*}
\end{gathered}
$$


where $F$ is an arbitrary complex function. In particular, setting $F=-\lambda=$ (const.) we obtain the nonlinear Schrödinger equation with power nonlinearity

$$
i \psi_{t}+\Delta \psi=\lambda|\psi|^{k} \psi
$$

We see that the cubic and quintic Schrödinger equations will belong to the above class of Galilei-similitude invariant equations. These type of nonlinear generalizations arise in applications as diverse as nonlinear optics, wave propagation in water, interactions of laser beams with plasma, turbulence and many others.

When we extend the Galilei invariant equation by

$$
D=x \partial_{x}+y \partial_{y}+2 t \partial_{t}
$$

we get the invariant equation corresponding to the realization $s g^{2}(0)$ of (2.12b):

$$
\begin{gathered}
i \psi_{t}+\Delta \psi+L_{2} F\left(S_{1}, S_{2}, S_{3}\right) \psi=0 \\
S_{1}=L_{1}, \quad S_{2}=\frac{L_{3}}{L_{2}}, \quad S_{3}=\frac{L_{4}}{L_{2}}=S_{2}^{*} .
\end{gathered}
$$

\subsection{The Schrödinger invariant equations}

To construct conformally invariant evolution equations we should add the requirement of conformal invariance generated by $C$. This will further restrict the form of the arbitrary functions occurring in the Galilei-similitude invariant equations. Indeed, for the standard realization $\operatorname{sch}^{1}(0 ; 0,0)$ the requirement that Eq. (3.8a) be invariant under

$$
C=x t \partial_{x}+y t \partial_{y}+t^{2} \partial_{t}+\left\{-2 / k t+i\left(x^{2}+y^{2}\right) / 4\right\} \psi \partial_{\psi}-\left\{2 / k t+i\left(x^{2}+y^{2}\right) / 4\right\} \psi^{*} \partial_{\psi^{*}}
$$

leads, after some lengthy calculations, to the invariant equation

$$
\begin{gathered}
i \psi_{t}+\Delta \psi+L_{1}^{k / 2}\left[\left(1-\frac{2}{k}\right) R_{3}+H\left(\Sigma_{1}, \Sigma_{2}\right)\right] \psi=0 \\
\Sigma_{1}=R_{2}=L_{1}^{-(k+4) / 2} L_{2}, \quad \Sigma_{2}=R_{1}+R_{3}=L_{1}^{-(k+4) / 2}\left(L_{3}+L_{4}\right) .
\end{gathered}
$$

where $H$ is an arbitrary complex function of two real variables. For $k=2$ equation (3.11) reduces to

$$
\begin{gathered}
i \psi_{t}+\Delta \psi+L_{1} H\left(\Sigma_{1}, \Sigma_{2}\right) \psi=0 \\
\Sigma_{1}=L_{1}^{-3} L_{2}, \quad \Sigma_{2}=L_{1}^{-3}\left(L_{3}+L_{4}\right) .
\end{gathered}
$$


Eq. (3.12) includes particularly the cubic Schrödinger equation

$$
i \psi_{t}+\Delta \psi=\mu|\psi|^{2} \psi \text {. }
$$

Using the identity

$$
L_{2}-\left(L_{3}+L_{4}\right)=|\psi|^{2} \Delta|\psi|^{2}
$$

yields

$$
\Sigma_{2}=\Sigma_{1}-\frac{\Delta|\psi|^{2}}{|\psi|^{4}}
$$

and so Eq. (3.12) can be expressed as

$$
i \psi_{t}+\Delta \psi+L_{1} H_{1}\left(\Omega_{1}, \Omega_{2}\right) \psi=0
$$

where $H_{1}$ is arbitrary and

$$
\Omega_{1}=\frac{(\nabla|\psi|)^{2}}{|\psi|^{4}}, \quad \Omega_{2}=\frac{\Delta|\psi|^{2}}{|\psi|^{4}} .
$$

In particular, when $H_{1}$ is restricted to be linear in $\Omega_{1}, \Omega_{2}$, namely

$$
H_{1}=A \Omega_{1}+B \Omega_{2}+C, \quad A, B, C \in \mathbb{C}
$$

then we obtain

$$
i \psi_{t}+\Delta \psi+|\psi|^{-2}\left(A(\nabla|\psi|)^{2}+B \Delta|\psi|^{2}+C|\psi|^{4}\right) \psi=0
$$

containing the following $\mathfrak{s c h}(2)$ invariant equations

$$
\begin{gathered}
i \psi_{t}+\Delta \psi=A \frac{(\nabla|\psi|)^{2}}{|\psi|^{2}} \psi, \\
i \psi_{t}+\Delta \psi=B \frac{\Delta|\psi|^{2}}{|\psi|^{2}} \psi
\end{gathered}
$$

and the cubic Schrödinger equation. It is clear that

$$
i \psi_{t}+\Delta \psi=B \frac{\Delta|\psi|}{|\psi|} \psi .
$$

belongs to the class (3.14). The nonlinear terms on the right side describe dissipative and diffusion processes in quantum mechanics. Also, setting $H_{1}=A\left(\Omega_{1}-\Omega_{2}\right)$ and using $\Delta \ln |\psi|=\Omega_{2}-\Omega_{1}$ we obtain

$$
i \psi_{t}+\Delta \psi=A(\Delta \ln |\psi|) \psi
$$


The modular class of nonlinear equations of (3.14) are time reversal invariant, namely invariant under the transformations $t \rightarrow-t, \quad \psi \rightarrow \psi^{*}$. Eq. (3.15d) was proposed as a stochastic interpretation of quantum mechanical vacuum dissipative effects [20]. Also, equations (3.15d)-(3.15d) fall into the class of equations introduced by Sabatier [6] and DG-equations [8]. In particular, these equations were shown to be linearizable for any $A \in \mathbb{R}[7]$. In passing, let us mention that for $A>1$ logarithmic Schrödinger equation (3.15d) admits a solitary wave solution propagating without deformation. A variety of equations proposed as mathematical models of quantum theory appear to be special cases of the Schrödinger invariant nonlinear equation (3.14).

Furthermore, if one specializes the arbitrary function $H_{1}$ in (3.12) to be

$$
H=A+B \Omega_{1}^{1 / 2}
$$

then Eq. (3.12) has the form

$$
i \psi_{t}+\Delta \psi+\left[A|\psi|^{2}+B\left(|\psi|_{x}^{2}+|\psi|_{y}^{2}\right)^{1 / 2}\right] \psi=0 .
$$

It is natural to call Eq. (3.16) two dimensional Eckhaus equation. One dimensional Eckhaus equation is defined to be

$$
i \psi_{t}+\psi_{x x}+\left(A|\psi|^{4}+B|\psi|_{x}^{2}\right) \psi=0
$$

where $A, B$ are arbitrary complex numbers. For $|B|^{2}=4 A$ with $A$ real, the above equation was shown to be linearizable, namely equivalent to the linear Schrödinger equation and to have the Painlevé property [21, 22]. It should be interesting to study the integrability and linearizability properties of (3.16).

In addition to Schrödinger invariance we can construct subsets of (3.12) satisfying the homogeneity condition. When this is the case we find that $H$ has the form

$$
H=\Sigma_{1} h\left(\frac{\Sigma_{1}}{\Sigma_{2}}\right) .
$$

On the other hand, the Galilei-similitude invariant equation (3.10) can not be extended to conformal transformations. This implies that there is no equation invariant under the realization (2.13c). However, notice that the arbitrary function in (3.10) can be specified so that equation satisfies homogeneity condition, namely

$$
i \psi_{t}+\Delta \psi+\frac{L_{2}}{L_{1}^{2}} F\left(S_{2}, S_{3}\right) \psi=0
$$

with $S_{2}, S_{3}$ as in (3.10). Let us comment that, here as opposed to the one dimensional case, no quintic Schrödinger equation invariant under Schrödinger group is obtained. Quintic Schrödinger equation is typical for one space dimension. 
Since all of the realizations obtained in section 2 generate fiber-preserving transformations, namely the coefficients multiplying $\partial_{x}, \partial_{y}$ and $\partial_{t}$ do not depend on $\psi, \psi^{*}$, in principle one can always obtain equations invariant under the less standard groups involving an arbitrary function of a single real variable $\psi \psi^{*}$ by integrating a system of coupled first order partial differential equations. But with this generality invariant equations are too complicated and we have not attempted to write them out here.

\section{Conclusions}

The results of this article can be summarized as follows. In section 2 we performed a classification of all possible realizations of the extended Galilei, Galilei-similitude and Schrödinger algebras in $2+1$ space-time dimensions in terms of vector field under local diffeomorphisms of $\mathbb{R}^{3} \times \mathbb{C}$. The realizations obtained depend on arbitrary functions of a real variable $\psi \psi^{*}$ and constants and the corresponding group transformations are necessarily fiber preserving.

In section 3 we constructed evolution type equations invariant under the groups corresponding to the realized algebras with the arbitrary functions set equal to zero. In other words, we obtained a variety of second order equations of the form (1.1) invariant under the symmetry group of the free Schrödinger equation, omitting the infinite dimensional Lie group that reflects linear superposition principle. Thus, we have shown that how general a Schrödinger invariant equation can be. In particular, we obtained a class of equations which might be candidates for possible generalizations of quantum mechanics for a scalar particle in $(2+1)$-dimensional space-time. Such general equations need a careful physical interpretation.

In the present study, as in [3], we obtain rather general invariant equations involving an arbitrary function of three or two variables (invariants). But, contrary to the $(1+1)$-dimensional case, we do not obtain quintic Schrödinger equation invariant under any realization of the Schrödinger group. Let us mention that some realizations of [3] do not have counterparts in three dimensional case and vice versa. One thing that is common to both dimensions is that all realized algebras involve an arbitrary function of a real variable, namely they characterize a class of algebras and that they correspond to fiber preserving transformations. The latter property simply means that the transformed independent variables under the action of the corresponding group will only depend on the old ones, but not on the wave function. When the arbitrary function figuring in the most general Schrödinger invariant equation of the standard realization is restricted to certain subfamilies we recovered several invariant equations which fit in the class proposed in Ref. [6, 7, 8]. Another special case is two dimensional analogue of the integrable Eckhaus equation that was first introduced in 21] and generalized in 22]. 
The knowledge that the equations are by construction invariant under spacetime symmetry groups ensures us that we can always apply the symmetry reduction method to find exact particular solutions called group invariant solutions. For example, a recent study 23 has been devoted to symmetries and solutions of the vector Schrödinger equation with cubic nonlinearity in two space dimensions. In order to be able to perform symmetry reduction systematically we need to know a classification of the subalgebras of the corresponding symmetry algebra into conjugacy classes, under the action of the symmetry group. The subalgebras of Schrödinger algebra $\mathfrak{s c h}(2)$ were classified by $\mathrm{G}$. burdet et al. in [14]. In a separate paper we plan to investigate a classification of symmetry reductions and their solutions for a specific class of invariant equations such as those of the form (3.15). Especially, we would like to return to the integrability properties of (3.16).

\section{Acknowledgement}

The author would like to thank the refrees for their useful comments which improved the presentation of the paper.

\section{References}

[1] G. Rideau and P. Winternitz. J. Math. Phys., 31(5):1095-1105, 1990.

[2] F. Güngör. J. Phys. A: Math. Gen., 31:697-706, 1998.

[3] G. Rideau and P. Winternitz. J. Math. Phys., 34(3):558-570, 1992.

[4] R.H. Heredero and P.J. Olver. J. Math. Phys., 37(12):6414-6438, 1996.

[5] W.I. Fushchich and I.A. Yegorchenko. Acta Appl. Math., 28:69-92, 1992.

[6] P.C. Sabatier. Inverse Problems, 6:L47-L53, 1990.

[7] G. Auberson and P.C. Sabatier. J. Math. Phys., 35(8):4028-4040, 1994.

[8] H-D. Doebner and G. A. Goldin. J. Phys. A: Math. Gen., 27:1771-1780, 1994.

[9] P. Nattermann and H-D. Doebner. J. Nonlin. Math. Phys., 3(3-4):302-310, 1996.

[10] S. Weinberg. Ann. Phys., 194:336-386, 1989.

[11] R.Z. Zhdanov and W.I. Fushchych. J. Nonlin. Math. Phys., 4(3-4):426-435, 1997.

[12] W.I. Fushchich and R.M. Cherniha. J. Phys. A: Math. Gen., 28:5569-5579, 1995. 
[13] J. Negro, M.A. del Olmo, and A. Rodriguez-Marco. J. Math. Phys., 38(7):37863809, 1997.

[14] G. Burdet, J. Patera, M. Perrin, and P. Winternitz. Ann. Sc. Math. Quebec, II(1):81-108, 1978.

[15] P.J. Olver. Applications of Lie Groups to Differential Equations. Springer, New York, 1991.

[16] P.J. Olver. Equivalence, Invariants and Symmetry. Cambridge University Press, Cambridge, 1995.

[17] A.O. Barut and R. Raczka. Theory of Group Representations and Applications. Polish Sci. Publ., Warsaw, 1980.

[18] L.V. Ovsiannikov. Group Analysis of Differential Equations. Academic Press, New York, 1982.

[19] G.W. Bluman and S. Kumei. Symmetries and Differential Equations. Springer, New York, 1989.

[20] J.P. Vigier. Phys. Lett. A, 135:99-105, 1989.

[21] F. Calogero and S. De Lillo. Inverse Problems, 4:L33-L37, 1988.

[22] P.A. Clarkson. Nonlinearity, 5:453-472, 1992.

[23] A. Sciarrino and P. Winternitz. Il Nuovo Cimento, 112B(6):853-871, 1997. 